\title{
Alterações visuais encontradas em uma amostra de caminhoneiros nas rodovias do Estado de São Paulo
}

\section{Visual alterations in a sample truck driver population in the highways of the state of São Paulo}

\author{
Eduardo Costa Sá ${ }^{1}$, Emilio Zuolo Ferro ${ }^{2}$, Felipe Fontana Alpert ${ }^{3}$, \\ Diego Toniolo Prado ${ }^{4}$, Daniel Romero Muñoz
}

DOI: http://dx.doi.org/10.11606/issn.2317-2770.v23i2p70-74

\begin{abstract}
Sá EC, Ferro EZ, Alpert FF, Prado DT, Muñoz DR. Alterações visuais encontradas em uma amostra de caminhoneiros nas rodovias do Estado de São Paulo. Saúde, Ética \& Justiça. 2018;23(2):70-4.

RESUMO: INTRODUÇÃO: a realização de exame de aptidão física e mental dos motoristas visa à identificação de doenças de risco para a segurança do trânsito, incluindo os transtornos visuais. A avaliação oftalmológica deve ser realizada de forma completa em todos os motoristas, e deve compreender a análise da acuidade visual, do campo visual, da visão cromática e o teste de ofuscamento, de acordo com a resolução 267/08 do CONTRAN. OBJETIVO: determinar a prevalência de alterações visuais em uma amostra de caminhoneiros abordados nas Rodovias Regis Bittencourt (BR116) e Fernão Dias (BR-381). MÉTODO: foi realizado estudo transversal com caminhoneiros por meio de avaliação oftalmológica com a utilização do Rizomed ${ }^{\circledR}$. RESULTADOS: foram avaliados 89 caminhoneiros. Quanto à acuidade visual, 19,1\% dos examinados apresentaram visão inferior a $0,66 \mathrm{em}$ ambos os olhos e $24,7 \%$ mostraram acuidade visual menor que 0,66 em um dos olhos e menor que 0,50 no outro olho e visão binocular menor que 0,80 . No que se refere à visão estereoscópica, 1,1\% evidenciaram percepção alterada de profundidade. Quanto à campimetria horizontal, 44,9\% apresentaram alteração de campo visual. Quanto ao ofuscamento, 12,4\% tiveram uma reação considerada alterada. Quanto à visão cromática $100 \%$ dos examinados conseguiram distinguir as cores verde, amarelo e vermelha. CONCLUSÕES: foram encontradas importantes alterações visuais em uma amostra de caminhoneiros abordados nas rodovias do Estado de São Paulo. Este achado leva à ideia de se propor um menor intervalo de tempo para a renovação da CNH dos motoristas das categorias C, D e E, incluindo uma consulta periódica ao médico oftalmologista.
\end{abstract}

DESCRITORES: Acuidade Visual; Testes Visuais; Exame para Habilitação de Motoristas.

\footnotetext{
1. Centro Universitário Saúde ABC (Faculdade de Medicina do ABC)

2. Residente de Medicina Legal e Perícias Médicas da Faculdade de Medicina da Universidade de São Paulo

3. Residente de Medicina do Trabalho da Faculdade de Medicina da Universidade de São Paulo

4. Residente de Medicina do Trabalho da Faculdade de Medicina da Universidade de São Paulo

5. Professor Titular do Departamento de Medicina Legal, Ética Médica e Medicina Social e do Trabalho da Faculdade de Medicina da Universidade de São Paulo

Endereço para correspondência: email: eduardocs6@gmail.com
} 


\section{INTRODUÇÃO}

$\mathrm{O}$ número de óbitos no mundo por acidentes no trânsito é preocupante. As estatísticas nacionais registraram 36.430 mortes no ano de $2017^{1}$. No Brasil, as mortes decorrentes de causas externas representam a terceira colocação entre as principais causas de óbitos. Ela passa a ser a primeira causa de morte quando se considera apenas a faixa etária dos 10 aos 39 anos $^{2}$.

A Associação Brasileira de Logística e Transporte de Carga afirma que o transporte de cargas é responsável por $25 \%$ dos acidentes ocorridos nas rodovias. E, quando comparado a outras atividades econômicas, responde sozinho por $15 \%$ dos óbitos nas rodovias e 7 $\%$ dos casos de invalidez permanente. Os acidentes com caminhões seguidos de óbito são responsáveis por $40 \%$ das ocorrências, conforme número da Policia Rodoviária Federal $^{3}$.

Evidências demonstram que motoristas profissionais que dirigem longas distâncias procuram menos assistência médica, e por isto seriam eles os responsáveis pela maior parte de acidentes fatais em rodovias ${ }^{4,5}$. Segundo relatório anual realizado pela Seguradora Líder, o DPVAT indenizou 13.165 motoristas de caminhões e pick-ups no ano de 2017 em decorrência de acidente seguido de morte ou invalidez ${ }^{6}$.

A realização de exame de aptidão física e mental dos motoristas de forma cuidadosa e periódica poderia não só identificar os portadores de doença de risco para a segurança do trânsito, como também afastá-los quando houver necessidade para tal'

De acordo com Adura ${ }^{7}$, as doenças orgânicas são responsáveis por $6,5 \%$ dos acidentes fatais. Para evitar o aumento desta taxa, os médicos peritos devem seguir à risca o Código de Trânsito Brasileiro (CTB), assim como a Resolução 425 de 27 de novembro de 2012 do Conselho Nacional de Trânsito (CONTRAN), que normatiza os procedimentos do exame de aptidão física e mental para condutores de veículos. Durante muitos anos esta avaliação médica foi chamada de "exame de vista", o que demonstra a importância da avaliação oftalmológica dos condutores na prevenção de acidentes?

Apesar de a maioria dos motoristas profissionais saber que a baixa acuidade visual está relacionada ao maior número de acidentes no trânsito, eles não se submetem a avaliação oftalmológica com frequência. É comum observar que grande parte dos motoristas profissionais com alterações visuais não usa correção adequada para as suas patologias .

As pesquisas relacionando visão e trânsito tiveram início em 1925, em Nova Jersey. Naquela época, era recomendado que os condutores de veículos motorizados tivessem uma acuidade visual de pelo menos 20/50 em um olho e pelo menos 20/100 no olho contralateral, com ou sem correção. O candidato com acuidade visual menor que 20/100 no olho pior poderia ser qualificado para conduzir um veículo motorizado desde que liberado por um conselho especial. Os portadores de diplopia não eram liberados para dirigir 9 .

$\mathrm{Na}$ Inglaterra os testes de função visual simples, sem avaliação da parte cognitiva, são feitos aos 17 anos, quando se tira a Carteira de Habilitação e, posteriormente, apenas aos 70 anos. Neste meio tempo, os candidatos estão legalmente liberados para dirigir, mesmo que tenham adquirido déficits visuais severos. Estudo realizado nesse país, por meio de questionários e testes aplicados aos motoristas na estrada aleatoriamente, observou a presença de muitas disfunções visuais importantes nos mesmos. Os autores desse estudo concluíram haver a necessidade não só de uma consciência individual dos motoristas, mas, também, uma responsabilidade do sistema público de evitar que isto aconteça, diminuindo o tempo de intervalo entre os testes de visão para que se diminua significativamente o número de acidentes ${ }^{10}$.

Partindo do princípio que os acidentes de trânsito podem ser provocados por inúmeros fatores, principalmente a baixa acuidade visual, a avaliação oftalmológica é de suma importância e deve ser realizada de forma completa, principalmente nos motoristas profissionais.

A avaliação oftalmológica, no Brasil, deve compreender a análise da acuidade visual, do campo visual, da visão cromática e do teste de ofuscamento, de acordo com a resolução 267/08 do CONTRAN.

A acuidade visual é a avaliação mais relevante do exame oftalmológico, segundo dados da OMS (2008). Para este exame são utilizadas escalas optométricas que devem ser lidas a uma distância de 6 metros (20 pés), sendo o resultado obtido correspondente à fração de 1/20 (tabela de SNELLEN). O primeiro valor da fração corresponde à distância do teste em pés entre a tabela de letras a serem lidas e o candidato e o segundo valor representa a fileira menor que o candidato é capaz de ler.

O valor normal é 20/20, o que significa que o olho do candidato pode ler a uma distância de 20 pés o que um olho normal também conseguirá ler. No caso de o candidato não conseguir ler todas as letras de uma fileira ele deverá ser considerado apto se conseguir acertar metade das letras mais uma. Quando houver incapacidade total de leitura das letras, outros testes deverão ser realizados, como o da capacidade de contar dedos, ou, ainda, o de detectar movimentos da mão ou o da percepção luminosa.

Segundo o anexo II da resolução 267/08 do CONTRAN (Anexo A), a acuidade visual aceita para as classes ACC, A e B é maior ou igual a 20/40 $(0,50)$ em cada olho ou maior ou igual a 20/30 $(0,66) \mathrm{em}$ um dos olhos com pelo menos percepção luminosa no outro olho. Para as classes C, D e E a acuidade visual aceita é 
maior ou igual a 20/30 $(0,66)$ em cada olho ou maior ou igual a 20/30 $(0,66)$ em um olho e maior ou igual a 20/40 no outro, com visão binocular mínima de 20/25 $(0,80)^{7}$.

A avaliação do campo visual pode ser feita de forma comparativa com o do médico examinador por meio da campimetria manual ou, ainda, utilizandose um aparelho que mede o campo visual horizontal, como o da marca Rizomed $\AA$. A campimetria horizontal é feita através da somatória, realizada pelo examinador, da visão nasal e temporal dos olhos separadamente. $\mathrm{O}$ Rizomed® pode ser utilizado para fazer este exame, já que ele indica a graduação em graus dos campos visuais analisados após o posicionamento da cabeça do motorista em ponto fixo indicado no aparelho e movimentação de pontos luminosos a uma angulação de até 180 graus.

Para as classes ACC, A e B, aceita-se um campo visual maior ou igual a $60^{\circ} \mathrm{em}$ cada um dos olhos ou maior ou igual a $120^{\circ} \mathrm{em}$ um olho. Para as classes C, D e E o campo visual deverá ser maior ou igual a $120^{\circ} \mathrm{em}$ cada um dos olhos?

A avaliação da visão cromática é feita avaliandose a distinção das cores verde, vermelho e amarelo pelo candidato. No Rizomed $\AA$, após a ativação do dispositivo das cores que precisam ser identificadas, os candidatos são solicitados a reconhecê-las, diferenciando-as de forma verbal para o examinador. Os candidatos de todas as classes devem ser capazes de distinguir estas três cores. Candidatos que sejam portadores de discromatopsias que consigam identificar estas três cores são considerados aptos para qualquer categoria, sem restrições ${ }^{7}$.

O teste de ofuscamento é feito através da identificação de letras pelo candidato após ofuscamento com duração breve. Este teste é realizado com a exposição do examinado à luminosidade de 20 a 40 lux por um tempo de 2 a 4 segundos. Após o apagamento da luz, o candidato precisa identificar o optotipo o mais rápido possível. Para ser aprovado em qualquer uma das classes, o candidato deverá possuir recuperação rápida após ofuscamento ${ }^{7}$.

A avaliação da visão de profundidade, também chamada de estereopsia, deveria ser feita em todos os candidatos para que se pudesse identificar portadores de estrabismo, ambliopia, nistagmo e visão monocular. Os portadores de estrabismo, mesmo com acuidade visual normal, não possuem visão estereoscópica, ou seja, não possuem noção de profundidade, razão pela qual os portadores desta patologia não são liberados para possuir habilitação das classes C, D e E. Os candidatos com ambliopia também não deveriam ser liberados para se habilitar nas classes $\mathrm{C}, \mathrm{D}$ e $\mathrm{E}$ devido à gravidade $\mathrm{e}$ irreversibilidade do quadro ${ }^{7}$. Apesar da importância deste teste para os candidatos às categorias $\mathrm{C}, \mathrm{D}$ e E da Carteira Nacional de Habilitação (CNH), não existe um item, no anexo II da resolução 267 do CONTRAN, que referende a visão estereoscópica.
Apesar das causas de acidentes serem multifatoriais e complexas, sabe-se que a função visual em bom estado é um pré-requisito essencial para a direção segura ${ }^{11}$.

Como os acidentes de trânsito envolvendo caminhoneiros são de extrema gravidade e repercussão na saúde pública, é necessário que haja trabalhos no intuito de verificar a presença de alterações oftalmológicas capazes de aumentar a prevalência de acidentes de trânsito nas rodovias de São Paulo.

\section{OBJETIVO}

Determinar a prevalência de alterações visuais em uma amostra de caminhoneiros abordados nas Rodovias Regis Bittencourt (BR-116) e Fernão Dias (BR-381) nos dias 17 e 24 de Agosto de 2011, respectivamente.

\section{MATERIAL E MÉTODO}

Foi realizado um estudo transversal com caminhoneiros que trafegaram nas Rodovias Regis Bittencourt e Fernão Dias no dia 17 de agosto em Itapecerica da Serra (entre as 8:00 e as 17:00 horas) e no dia 24 de agosto na Fernão Dias km 30 (das 13:00 às 19:00 horas), respectivamente.

Foi realizada a avaliação oftalmológica com o aparelho Rizomed $\AA$. Os dados coletados foram tabulados no programa Microsoft Office Excel 2007 e, posteriormente, foi feita a análise estatística, tendo como referência os valores normatizados pela Resolução 267/08 do CONTRAN para as categorias C, D e E da CNH.

De acordo com o anexo II da Resolução 267/2008 do CONTRAN, para as categorias C, D e E da Carteira Nacional de Habilitação (CNH), a acuidade visual deverá ser:

- igual ou superior a 20/30 (equivalente a 0,66 ) em cada um dos olhos OU

- igual ou superior a 20/30 (equivalente a 0,66) em um olho e igual ou superior a 20/40 (equivalente a $0,50)$ no outro, com visão binocular mínima de 20/25 (equivalente a 0,80 ).

Os caminhoneiros inicialmente responderam, de forma oral, a duas perguntas: qual a sua idade e quando foi sua última consulta ao oftalmologista. A partir de então, foram submetidos à avaliação oftalmológica com as lentes corretivas, quando necessárias.

Todos os exames foram realizados por dois

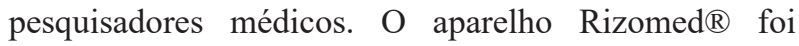
utilizado para avaliar a acuidade visual, a visão estereoscópica, a visão cromática, o ofuscamento, a visão noturna (adaptometria) e o campo visual.

\section{RESULTADOS}

Em uma amostra de 89 pessoas do sexo masculino, 
a média de idade foi de 43,8 anos, sendo a mediana de 43 anos e o desvio padrão de 12,7 anos.

Referente ao questionamento sobre o tempo decorrido desde a última consulta ao oftalmologista, a média foi de 17 meses, com desvio padrão de 8 meses. Porém, 22,5\% dos motoristas avaliados afirmaram nunca ter ido ao oftalmologista.

Quanto à acuidade visual, 19,1\% dos examinados apresentaram visão inferior a $0,66 \mathrm{em}$ ambos os olhos e $24,7 \%$ possuíram acuidade visual menor que 0,66 em um dos olhos e menor que 0,50 no outro olho e visão binocular menor que 0,80 .

Do total avaliado, quanto à campimetria horizontal, $44,9 \%$ tiveram campo visual menor que 120 graus em cada um dos olhos, ou seja, apresentaram alteração de campo visual.

Quanto ao ofuscamento, 12,4\% dos caminhoneiros tiveram um tempo de reação maior que 2 segundos, ou seja, considerado alterado de acordo com o manual do proprietário do Rizomed $\AA$.

No que se refere à visão estereoscópica, $1,1 \%$ dos caminhoneiros estudados possuíram percepção alterada de profundidade.

Quanto à visão cromática $100 \%$ dos examinados conseguiram distinguir as cores verde, amarela e vermelha.

\section{DISCUSSÃO}

No Brasil, o conhecimento sobre o desempenho dos motoristas e a relação da acuidade visual dos mesmos tem crescido, dada a importância de prevenir acidentes e, consequentemente, as mortes no trânsito.

Johnson e Wilkinson ${ }^{12}$, no seu estudo Vision and Driving: The United States (1998), demonstraram que há correlação entre o bom desempenho do motorista com as medidas de acuidade visual e campo visual, durante as suas medições nas avaliações americanas.

Estudo realizado na Inglaterra em 2007, por meio de questionários e testes aplicados aos motoristas na estrada aleatoriamente, observou a presença de muitas disfunções visuais importantes nos mesmos ${ }^{10}$.

Em nosso estudo, demonstramos a frequente prevalência de transtornos visuais nos motoristas das estradas do Estado de São Paulo. Dentre os critérios avaliados para a obtenção de $\mathrm{CNH}$ para as categorias $\mathrm{C}$, D e E, a alteração da campimetria horizontal foi a mais observada, atingindo 44,9\% dos motoristas. As alterações da acuidade visual foram encontradas em $24,7 \%$ dos avaliados.

Porém, no estudo realizado por Keay et al. entre 2005 e 2007, nos EUA, a perda de campo visual e da atenção visual não apresentaram associação estatística com o mau desempenho dos condutores nas estradas ${ }^{13}$.

Os dados desta pesquisa não nos permitem extrapolar para a população em geral, porém, acredita-se, devem ser realizados outros estudos semelhantes e com maior amostragem, permitindo fazer inferências quanto a este importante tema de saúde pública.

\section{CONCLUSÃO}

Foram encontradas importantes alterações na avaliação da acuidade visual, do campo visual, da noção de profundidade e do teste de ofuscamento em uma amostra de caminhoneiros abordados nas rodovias do Estado de São Paulo. Este achado leva à ideia de se propor um menor intervalo de tempo para a renovação da $\mathrm{CNH}$ dos motoristas das categorias $\mathrm{C}, \mathrm{D}$ e $\mathrm{E}$, incluindo uma consulta periódica ao médico oftalmologista.

Sá EC, Ferro EZ, Alpert FF, Prado DT, Muñoz DR. Visual alterations in a sample truck driver population in the highways of the state of São Paulo. Saúde, Ética \& Justiça. 2018;23(2):70-4.

\begin{abstract}
The physical and mental examination of drivers checks for diseases that pose a safety risk to road users, including visual disorders. According to Resolution 267/08, from the Brazilian National Traffic Council (CONTRAN), a comprehensive visual test must be conducted, assessing visual acuity, field of vision, color vision and glare sensitivity. OBJECTIVE: To determine the prevalence of visual alterations in a sample population of truck drivers. Data was collected at Highways Regis Bittencourt (BR-116) and Fernão Dias (BR-381). METHOD: We conducted a cross-sectional study on truck drivers through the Rizomed ${ }^{\circledR}$ ophthalmology evaluation. RESULTS: 89 truck drivers were analyzed. Regarding visual acuity, $19.1 \%$ were bellow 0.66 in both eyes and $24.7 \%$ were bellow 0.66 in one eye and bellow 0.50 in the other eye and had binocular vision inferior to 0.80 . Regarding stereoscopic vision, $1.1 \%$ showed alterations in depth perception. The horizontal campimetry showed $44.9 \%$ had alterations in their visual field. Regarding glare sensitivity, $12.4 \%$ presented altered reaction. Regarding color vision, $100 \%$ were capable of distinguishing the colors green, yellow and red. CONCLUSIONS: Significant visual alterations were found among the sample group of truck drivers in the highways of the state of São Paulo. This finding suggests that a shorter interval for renewing the driver's license in categories $\mathrm{C}, \mathrm{D}$ and $\mathrm{E}$ (which allow driving heavy motor vehicles) would be appropriate, including periodic consultation with an ophthalmologist.
\end{abstract}

KEY WORDS: Visual Acuity; Vision Tests; Automobile Driver Examination. 


\section{REFERÊNCIAS}

1) Brasil. Ministério da Saúde. Portal da Saúde [Homepage na Internet]. Brasília; 2017 [acesso em 2018 fev. 05]. Disponível em: http://www2.datasus.gov.br/DATASUS/ index.php? area $=0205 \& \mathrm{id}=6940 \& \mathrm{VObj}=\mathrm{http}: / /$ tabnet. datasus.gov.br/cgi/deftohtm.exe?sim/cnv/ext10

2) Brasil. Ministério da Saúde. Secretaria de Vigilância em Saúde. Vigilância de violências e acidentes em serviços de urgência e emergência. Viva Inquérito - Capitais e Distrito Federal. Brasil, 2011. Boletim Epidemiológico [Internet]. 2013 [acesso em 2018 fev. 05]; 44(8):1-7. Disponível em: http://portalarquivos2.saude.gov.br/images/pdf/2014/ junho/11/BE-2013-44--8----VIVA-Inquerito.pdf Vol. 44 $n^{0} 8$ - 2013 -

3) Silva, LG. O trabalho dos motoristas de caminhão: a relação entre atividade, vínculo empregatício e acidentes de trabalho [Dissertação]. São Paulo: Faculdade de Saúde Pública, Universidade de São Paulo; 2011.

4) Austin M. Clinic keeping truckers tuned up. Truck stop offers health on the road. Colo Nurse. 2006;106(2):19.

5) Solomon AJ, Doucette JT, Garland E, McGinn T. Healthcare and the long haul: Long distance truck drivers--A medically underserved population. Am J Ind Med. 2004;46(5):46371. DOI: http://dx.doi.org/10.1002/ajim.20072

6) Seguradora Líder. Administradora do Seguro DPVAT Relatório Anual Seguradora Líder-DPVAT 2017 [Internet] Rio de Janeiro; 2017 [acesso em 2018 fev. 08]. Disponível em: https://www.seguradoralider.com.br/Documents/
Relatorio-Anual/Relatorio-Anual-Seguradora $\% 20$ Lider_2017.pdf

7) Adura FE, Sabbag AF. Manual para o médico perito examinador de candidatos a motorista. $4^{\mathrm{a}}$ ed. São Paulo: Abramet; 2012.

8) Ovenseri-Ogomo G, Adolfo M. Poor vision, refractive errors and barriers to treatment among commercial vehicle drivers in the Cape Coast municipality. Afr Health Sci. 2011;11(1):97-102.

9) Kotecha A, Spratt A, Viswanathan A. Visual function and fitness to drive. Br Med Bull. 2008;87:163-74. DOI: http:// dx.doi.org/10.1093/bmb/ldn028

10) Anuradha S, Potter C, Fernquest G. Vision and drivers - a South Wales survey. JPublic Health(Oxf). 2007;29(3):230-5. DOI: http://dx.doi.org/10.1093/pubmed/fdm031

11) Yazdan-Ashoori P, Ten Hove M. Vision and driving: Canada. J Neuro-Ophtalmol. 2010;30(2):177-85. DOI: http://dx.doi. org/10.1097/WNO.0b013e3181dfa982

12) Wilkinson ME. Driving with a visual impairment. J Am Soc Ophthal Registered Nurses, Insight. 1998;23(2):48-52.

13) Keay L, Munoz B, Turano KA, Hassan SE, Munro CA, Duncan DD, et al. Visual and cognitive deficits predict stopping or restricting driving: the salisbury eye evaluation driving study (SEEDS). Invest Ophthalmol Vis Sci. 2009;50(1):107-13. DOI: http://dx.doi.org/10.1167/ iovs.08-2367

Recebido em: 17/06/2018

Aceito em: 25/08/2018 